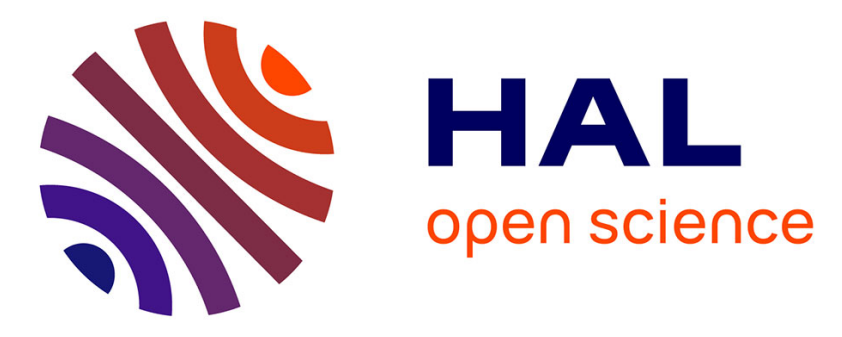

\title{
Fast image deconvolution for enhancement of the resolution in the video rate terahertz imaging
}

Arash Rashidi, Amalya Minasyan, Alexis Cailly, Maher Hamdi, Olivier

Redon, Laurent Dussopt, Hussein Yahia

\section{- To cite this version:}

Arash Rashidi, Amalya Minasyan, Alexis Cailly, Maher Hamdi, Olivier Redon, et al.. Fast image deconvolution for enhancement of the resolution in the video rate terahertz imaging. IRMMW-THz, 46th International Conference on Infrared, Millimeter, and Terahertz Waves, Aug 2021, Beijing, China. hal-03343739

\author{
HAL Id: hal-03343739 \\ https://hal.inria.fr/hal-03343739
}

Submitted on 14 Sep 2021

HAL is a multi-disciplinary open access archive for the deposit and dissemination of scientific research documents, whether they are published or not. The documents may come from teaching and research institutions in France or abroad, or from public or private research centers.
L'archive ouverte pluridisciplinaire HAL, est destinée au dépôt et à la diffusion de documents scientifiques de niveau recherche, publiés ou non, émanant des établissements d'enseignement et de recherche français ou étrangers, des laboratoires publics ou privés. 


\title{
Fast image deconvolution for enhancement of the resolution in the video rate terahertz imaging
}

\author{
A. Rashidi ${ }^{1,2}$, A. Minasyan ${ }^{2}$, A. Cailly ${ }^{2}$, M. Hamdi ${ }^{3}$, O. Redon ${ }^{3}$, L. Dussopt ${ }^{3}$, H. Yahia ${ }^{1}$ \\ ${ }^{1}$ INRIA Geostat, Talence, 33405, France \\ ${ }^{2} \mathrm{I} 2 \mathrm{~S}$, Pessac, 33608, France \\ ${ }^{3}$ CEA Tech Nouvelle-Aquitaine, Pessac, 33600, France
}

\begin{abstract}
A fast image deconvolution algorithm is used to demonstrate the resolution enhancement of video rate camera acquired Terahertz images. Our algorithm is based on variable splitting technique with the use of a family of sparsity inducing regularizers for the first time in an image deconvolution application, it is also suitable for practical applications in industry with computationally constrained conditions. The results of the proposed process provide substantial enhancement on the quality and resolution of THz images.
\end{abstract}

\section{INTRODUCTION}

$\mathrm{T}$ ERAHERTZ (THz) imaging has been developing very fast recently. Among various imaging techniques, camera technology has its advantages in terms of speed, bandwidth, and ease of use. I2S TZcam camera is based on CEA-LETI $320 \times 240$ antenna-coupled bolometer array and has demonstrated state-of-the-art performances for real-time imaging at $0.1-5 \mathrm{THz}[1-2]$. However, the resolution of the images is often limited by the characteristics of the material traversed as well as by the wavelength of the $\mathrm{THz}$ illumination.

Many research works are devoted to the resolution enhancement in the far field of $\mathrm{THz}$ imaging through deconvolution techniques [3], which is a widely studied and challenging research area in the field of image processing.

In this work, we propose a new fast image deconvolution algorithm using approximated proximal operators. It combines the idea of variable splitting [4] with new sparsity inducing regularizers. For a THz imaging system, the image processing can be mathematically modeled in the matrix formation by reconstructing an image $x$ from an indirect blurred and noisy observation $y$ :

$$
y=H x+n \quad \text { (1) }
$$

where $H$ denotes a known PSF in matrix form and $n$ additive Gaussian noise with zero mean and known variance $\sigma^{2}$. A simple and common approach to solve the problem is using regularization methods i.e., by solving the following optimization problem:

$$
\arg \min \frac{\mu}{2}\|H x-y\|^{2}+\varphi(D x)
$$

In our case $D$, denotes the discrete gradient operator. The first term of the equation measures the closeness of solution to the observation $y$, it is known as data fidelity term. The second term $\varphi(u)$, the regularizer, enforces a prior knowledge about $x$ into the solution.

\section{PROPOSED METHOD AND APPROACH}

In this section, we will briefly explain the technique to minimize the optimization problem of (2). Here we briefly show the implementation of variable splitting. Variable splitting is a very simple procedure that consists of creating a new variable, say $u$, to serve as the argument of $\varphi($.$) under$ the constraint that $u=D x$. The idea is to consider the constrained problem:

$$
\operatorname{argmin}_{x, u} \frac{\mu}{2}\|H x-y\|^{2}+\varphi(u) \text { s.t } u=D x
$$

Given the constraint, it must be that $u=D x$, so the solution to equations (3) and (2) must be the same or very close. In order to enforce the constraint, we will add an additional term to the cost function that penalizes large differences between $u$ and $D x$ This then results in the unconstrained optimization problem given by:

$$
\operatorname{argmin}_{x, u} \frac{\mu}{2}\|H x-y\|^{2}+\varphi(u)+\frac{\beta}{2}\|D x-u\|^{2}
$$

where $\beta$ is a constant that controls the gain of the penalty term. It also enforces the difference between $D x$ and $u$ to be as small as possible.

The problem formulated in the form of Eq. (3) can be solved by an alternating minimization scheme, i.e., solving two subproblems iteratively:

$$
\begin{gathered}
(S 1) \operatorname{argmin}_{u} \varphi(u)+\frac{\beta}{2}\|D x-u\|^{2} \\
(S 2) \operatorname{argmin}_{x} \frac{\mu}{2}\|H x-y\|^{2}+\frac{\beta}{2}\|D x-u\|^{2}
\end{gathered}
$$

An advantage of this formulations is that Fast-Fourier Transform can be used to solve (6) to reduce computational complexity.

\section{GENERAL FRAMEWORK AND ALGORITHM}

Our algorithm allows the use of multiple regularizers as formulated below:

$$
\varphi_{q}^{p}(x)= \begin{cases}\frac{1}{q}\left(1-\left(|x|^{p}+1\right)^{-q}\right) & q \neq 0 \\ \log _{e}\left(|x|^{p}+1\right) & q=0\end{cases}
$$

Their approximate proximal operators of (7) are provided as shrinkage/thresholding operators [5]:

$\operatorname{prox}_{\frac{1}{\beta}}^{\varphi_{q}^{p}(u)}=\left\{\begin{array}{cc}\left(1-\frac{p}{\beta}\left(\frac{|u|^{p-2}}{\left(|u|^{p}+1\right)^{q+1}}\right)\right)_{+} u & ,|u|>\eta_{1 / \beta} \\ 0, & \text { otherwise }\end{array}\right.$

Here $(x)_{+}$denotes the positive part of $x$. As shown by this equation, it is computed by a simple closed-form expression for values bigger than $\eta_{1 / \beta}$ and set to zero if smaller. Proximal 
operators work under extremely general conditions and they are fast which is suitable in industry with limited computation capability.

Depending on the value of $p$ and $q$, equation (7) defines a potential function, each function has its own characteristic and can be used as prior information in second term of equation (2). In Fig.1 we have depicted various forms of $\varphi_{q}^{p}(x)$.

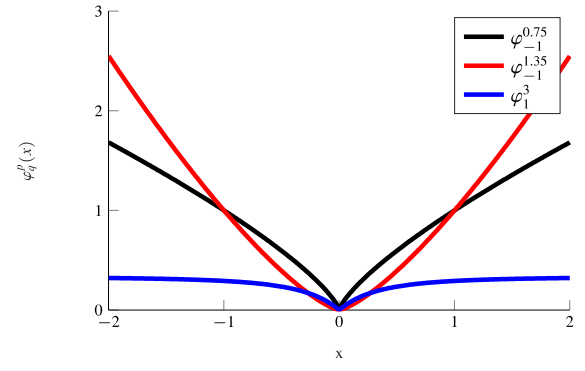

Fig. 1: Potential function for different values of $p$ and $q$.

We now give the overall algorithm using the general framework for the sub-problem (S1). As outlined in Algorithm 1 below, we minimize Eq. (4) by solving the $u$ and $x$ subproblems separately until the algorithm converges. We keep $\beta$ constant $(\beta=256)$ which makes our algorithm simpler and reduces computational complexity.

In step 2 of the algorithm, the solution of subproblem (S1) of equations (5) is calculated using the approximate proximal operator of equation (8).

On step 3 the $u$ obtained from step 2 is used to calculate the $x$ of Sub problem (S2) of equation (6). This process is repeated until the algorithm is converged.

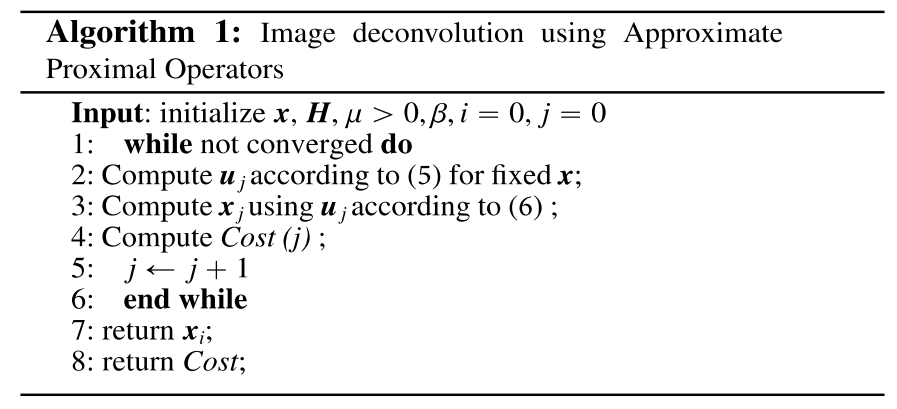

\section{RESULTS}

We begin by conducting a numerical experiment to quantify the loss in accuracy by using approximated Proximal Operators for image deconvolution using lena and camera man images. The solution of (4) is computed by two methods: the exact solution, as proposed in [6] and its approximation in (8) for some $\mathcal{L}_{p}=\varphi_{-1}^{p}(x)$ quasi-norms. We note that the approach of [6] has very high computational complexity in comparison to approximated Proximal Operators, not suitable for practical applications.

Since the difference cannot be assessed visually, In Table 1 we show the values of Mean Squared Error (MSE) for various values of $p$. The MSEs considered here are computed: I) Between the approximated solution and the original image.
II) Between the exact solution and the original image.

The closeness of solutions demonstrates that the use of approximated thresholding operators is almost indistinguishable from exact solution.

\begin{tabular}{|c|c|c|c|c|}
\cline { 2 - 5 } \multicolumn{1}{c|}{} & \multicolumn{2}{c|}{ Lena } & \multicolumn{2}{c|}{ Cameraman } \\
\hline$\ell_{p}$ & Exact & Approx & Exact & Approx \\
\hline$\ell_{3 / 4}$ & 0.0024 & 0.0024 & 0.0035 & 0.0035 \\
\hline$\ell_{1 / 2}$ & 0.0025 & 0.0025 & 0.0036 & 0.0036 \\
\hline$\ell_{1 / 3}$ & 0.0027 & 0.0027 & 0.0041 & 0.0040 \\
\hline
\end{tabular}

Table 1: Accuracy of approximation. The difference between approximated and exact solutions is negligible.

For a visual demonstration of deconvolution, we used various images at different frequencies acquired with TZcam of I2S. The results of deconvolution on THz images at 0.97 $\mathrm{THz}$ and $2.5 \mathrm{THz}$ is shown in Fig. 1. The time of the experiment for 10 iterations is 54 milliseconds on $\mathrm{C}++$ (CPU: Intel(R) Core i7 $3.2 \mathrm{GHz}$ ). A significant enhancement can be visually assessed. In the enhanced images, shapes and lines can be distinguished easily and the noise level is reduced notably.
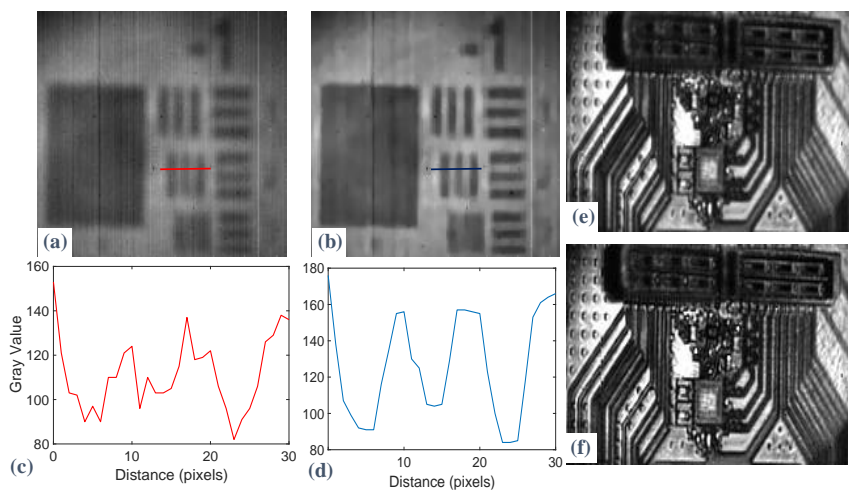

Fig. 1. (a) THz image of a $1951 \mathrm{USAF}$ resolution test chart at $0.97 \mathrm{THz}$ acquired with TZcam and x0.25 magnification lens. (b) Deconvolved image with $\varphi_{-1}^{0.7}$. (c) Plots of the Line Pairs pixel in grey values of the group-1 element 2 of the chart of original $\mathrm{THz}$ image, (d) deconvolved image. (e) Sample industrial application at $2.5 \mathrm{THz}$. (f) Deconvolved image of (e) with $\varphi_{-1}^{1}$.

\section{SUMMARY}

A fast deconvolution algorithm based on variable splitting technique with family of sparsity inducing regularizes was proposed and used on $\mathrm{THz}$ camera acquired images. The proposed method demonstrated notable enhancement of the resolution and the quality of $\mathrm{THz}$ images in various frequencies.

\section{REFERENCES}

[1]. "i2s - TZcam camera." [Online]. https://www.i2s.fr/en/product/tzcam . [2]. J. Meilhan et al., "Performance improvements of THz imagers based on uncooled antenna-coupled bolometer," 43rd Int. Conf. on Infrared, Millimeter and Terahertz Waves (IRMMW THz-2018), 9-14 Sept. 2018, Nagoya, Japan. [3]. Ning, Wei, et al. "Resolution enhancement in terahertz imaging via deconvolution." IEEE Access 7 (2019): 65116-65121.

[4]. Teodoro, Afonso M., José M. Bioucas-Dias, and Mário AT Figueiredo. "Image restoration and reconstruction using variable splitting and classadapted image priors." 2016 IEEE International Conference on Image Processing (ICIP). IEEE, 2016

[5] Gholami, Ali, and S. Mohammad Hosseini. "A general framework for sparsity-based denoising and inversion." IEEE transactions on signal processing 59.11 (2011): 5202-5211.

[6] Lorenz, D.A., 2007. Non-convex variational denoising of images: Interpolation between hard and soft wavelet shrinkage. Current Development in Theory and Application of Wavelets, 1(1), pp.31-56. 